\title{
RELATIONSHIP BETWEEN SCAPULAR MUSCLE PERFORMANCE AND GRIP STRENGTH IN LATERAL EPICONDYLITIS AMONG COM- PUTER OPERATORS
}

\author{
Priya S ${ }^{1}$, Harish S Krishna ${ }^{2}$, Theertha K ${ }^{* 3}$, Salbin Sebastian ${ }^{4}$. \\ ${ }^{1}$ Associate professor, Department of physiotherapy, Laxmi Memorial College of physiotherapy, \\ Mangalore, Karnataka, India. \\ 2 Professor Department of physiotherapy, Laxmi Memorial College of physiotherapy, Mangalore, \\ Karnataka, India. \\ ${ }^{*}$ Post graduate, Department of physiotherapy, Laxmi Memorial College of physiotherapy, Mangalore, \\ Karnataka, India. \\ ${ }^{4}$ Post graduate Department of physiotherapy, Laxmi Memorial college of Physiotherapy, Mangalore, \\ Karnataka, India.
}

\section{ABSTRACT}

Background: Lateral epicondylitis is an overuse injury of the tendon and involving the extensor muscle of forearm. Induced pain at the upper trapezius (UT) produces an increase in wrist extensor electromyographic activity (EMG) which could potentially lead to overuse injury, such as LE, at the elbow. Some studies found that there is a relation between scapular muscle muscle performance and lateral epicondylitis and loss of grip strength in lateral epicondylitis. There is a lack of literature of similar studies among computer operators. The most common wrong working posture in computer operator is excessive wrist extension it causes to the overuse injury like lateral epicondylitis.

The context and purpose of the study: To find out the relationship between scapular muscle performance and grip strength in lateral epicondylitis among computer operators.

Result: The correlation between scapular muscle performance and grip strength in LE among computer operators was done using Karl Person correlation coefficient. The result indicated that there is a significant relation between scapular muscle performance and grip strength in lateral epicondylitis.

Conclusion: The study showed that there is an impairment of scapular muscle performance and grip strength in lateral epcondylitis among computer operators.

Implication: Regular physical activity and proper ergonomics advice can improve quality of life among computer operators.

KEY WORDS: Scapular Muscle Performance, Grip Strength, Lateral Epicondylitis.

Address for correspondence: Dr. Theertha K, PT Post graduate, Department of physiotherapy, Laxmi Memorial College of physiotherapy, Mangalore, Karnataka, India.

E-Mail: theerthasanthosh256@gmail.com

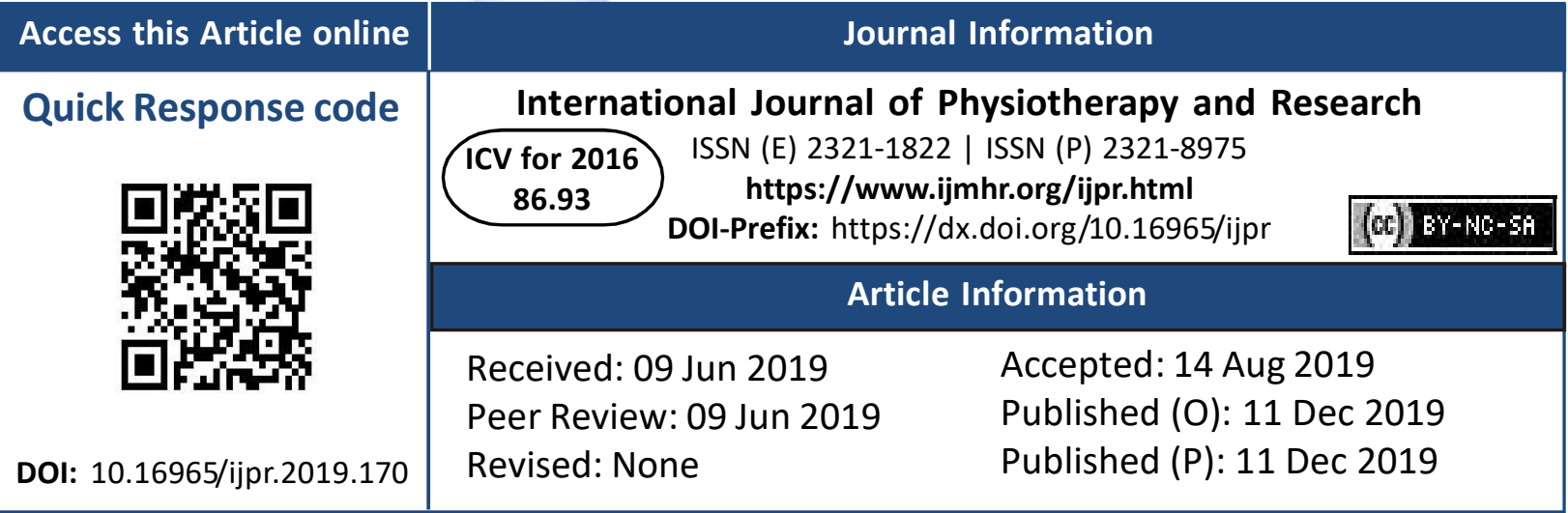

\section{BACKGROUND}

Lateral epicondylalgia (LE), as called lawn tennis elbow is characterized by pain in the lateral epicondyle of the humerus. Repetitive trauma caused by the overuse or overstresses of the wrist extensor of the forearm causes lateral epicondylitis. As related to the occupation the prevalence of tennis elbow is reported to be as 
high as $12.2 \%$. It mainly occurs after minor and often unrecognized trauma of the extensor muscle of the forearm and is characterized by insidious pain typically attributed to repetition of one event or activity, and also is considered to be an overload injury [1].

Samani A,et al found that induced pain at the upper trapezius (UT) produces an increase in wrist extensor electromyographic activity (EMG) which could potentially lead to overuse injury, such as LE, at the elbow. Musculoskeletal complaints during computer work, including portable computer, are caused by prolonged wrong body posture. The most common wrong working postures observed in computer operators are forward bending/ inclination of the back and head as well as excessive wrist extension. . The latter is especially important because bad wrist posture during work tasks is recognized as a risk factor in wrist and hand pain [2].

Maintaining wrist in extension is mainly achieved by the wrist extensor muscles, which is primarily and chiefly required in carrying out all the daily activities. Intrinsic factors can include altered joint arthro kinematics, muscular imbalance or muscular weakness in the upper extremity. Extrinsic factors can include errors in technique, environmental conditions, and equipment that alters the external forces applied to the upper extrimiy [3]. Pain over the lateral humeral epicondyle which radiates to the forearm, may get increased during excessive, quick, repetitive activities involving the hand in gripping or manipulating an object is the primary clinical manifestation So in this condition daily activities of the subject is adversely affected Both intrinsic and extrinsic factors contributes to the injury. Main complaint of the subject will be pain and decreased function, daily activities of the life like holding tools, shaking hands, lifting up a cup of coffee, dressing, household works, hitting a backhand stroke in tennis etc are affected.

The pathophysiology of Lateral epcondylitis is multi dimensional, it suggests that tendon cellular matrix changes may be accompanied by alterations in nociceptive processing and impairments in sensory and motor function [4]. The histological features of Lateral epicondylitis are similar to those of other common tendinopathies.
It includes increased cellularity, accumulation of ground substances, collagen disorganization and neurovascular in growth. Deep and anterior fibers of the extensor carpi radialis brevis (ECBR) component of the common extensor tendon origin are the most common site of focal degeneration.

Computer professionals who work on computer for long hours continuously, person may notice increasing aches and pains in some parts of the body, usually musculoskeletal in nature. Pain in the neck has become one of the leading problems nowadays [5] most of the epidemiological studies reviewed have defined repetitive work for the neck as work activities which involve continuous arm and hand movements which affect the neck/shoulder musculature and generate loads on the neck/shoulder area resulting in altered scapular position.

Several prospective studies have found that there is an associations between computer work and incidence of upper-limb pain. Patients with pain in the lateral epicondyle showed that increased pain and decreased grip strength due to the weakening of the rotator cuff and the scapula muscular systems Functional impingements of the shoulder joints occur because of the excessive compensation of the extensor resulting from impaired shoulder stability attributable to changes in the joint dynamics and muscle imbalance [6]. As a result, the symptoms of lateral epcondylitis appear because of minute damage to the elastic tissues. Overload due to repetitive forearm movements leads to changes in the shoulder complex and the symptoms of lateral epicondylitis appear because of the resultant forearm and hand muscle overload.

There are literatures on the relation between the scapular muscle performance and lateral epicondylities in general population, and some study found that the relationship between the grip strength and lateral epicondylitis, but there are no study available especially regarding the computer operators who are prone to develop this condition due to their work demand and working environment. Because of this necessary to find out the relation between scapular muscle performance and grip strength in lateral epicondylitis among computer operators. 


\section{MATERIALS AND METHODS}

This cross-sectional study was conducted on lateral epicondylitis among computer operators in Dakshina Kannada Mangalore. A total population of 30 samples was collected according to the sample size calculation from the previous article (JOSEPH M. DAY et al) Using the formula:

$$
\frac{n=Z \alpha^{2} \text { or }}{d^{2}}
$$

with an age group between $25-45$ years including both the genders. Subject were excluded from the study if they reported any of the following as part of their medical history: peripheral neuropathy secondary to diabetes, pro-gressive neurological disorder, cancer, infection in the spine or upper extrem-ity, upper motor neurological disorder (eg, stroke, traumatic brain injury), or fi-bromyalgia. Ethical clearance was obtained from the Institutional Ethical Committee. Subjects fulfilling the inclusion and exclusion criteria were enrolled for the study. Prior to the beginning of testing, the purpose and procedures of the study was explained to all participants and provided written informed consent.

Subject was recruited from tertiary hospital computer operators from manglore. Subject was recruited to participate in this study if they were seeking medical attention from a therapist and presented with at least 2 positive clinical tests for LE. The clinical tests performed for symp-toms of LE were palpation of the lateral epicondyle and the Mill's sign, Cozen's sign, and Maudsley's test. The tests were positive if there was reproduc-tion of pain at the lateral epicondyle.

\section{TECHNIQUE OF APPLICATION}

Scapular muscle endurance: The subject was prone lying will asked to place his or her forehead on the contra lateral limb. The limb should be positioned to $135^{\circ}$ of shoulder abduction. A $1 \%$ of weight cuff the participant's body weight will be strapped just to the elbow proximally. The subject will be then asked to elevate and hold his or her arm to the established level A single trial was performed and its value used for statistical analysis.

Strength of middle trapezius (MT), Upper trapezius (UT), Serratus anterior (SA) will measured by push pull dynamometer.

Strength of MT: subject was positioned in prone with their 90 degrees of shoulder abduction, with the shoulder in lateral rotation. The scapula was positioned in adduction. subject was maintain to their arm position as the therapist applied a downward force with the dynamometer over the distal third of the radial forearm until the subject maximal effort was overcome.

Strength of LT: Subject was positioned prone with a towel roll under their forehead. The extremity being tested was positioned diagonally overhead, in line with the muscle fibers and the scapula was passively positioned in an adducted and depressed position. The therapist provided manual fixation just inferior to the contra lateral scapula. subject was asked to maintain the arm position. The examiner provided pressure with the dynamometer in a downward direction over the distal third of the radial forearm until the subject 'maximal effort was overcome.

Strength of SA: The SA was tested by positioning the subject supine with the shoulder and elbow flexed to $90^{\circ}$. The dynamometer was placed on the olecranon of the el-bow. The subject was asked to protract the scapula, and resistance was given along the humeral axis [7].

Grip strength: grip strength was measurements by using hand held dynamometer with the elbow in 90 degrees of flexion for the affected extremity [8].

Statistical analysis: Analysis is done by descriptive statistic. correlation will be done by using Karl Pearson correlation coefficient test( $r$ ) . A statistical package SPSS version 17.0.will be used to do the analysis $p<.05$ will be considered as significant.

\section{RESULT}

Data were analyzed by the statistical program for social science (SPSS). Karl Pearson's correlation coefficient was used to check the relationship between scapular muscle performance and elbow positional grip strength in lateral epicondylitis among computer operators. The level of significance is less than 0.05 .

Descriptive Statistics were used to analysis the data obtained form the study. 
Table 1: The descriptive statistics of the Scapular muscle strength of $M T, L T$, and $S A$, endurance, disability in right side, EFR.

\begin{tabular}{|c|c|c|c|c|c|}
\hline & N & Minimum & Maximum & Mean & $\begin{array}{c}\text { Std. } \\
\text { Deviation }\end{array}$ \\
\hline AGE & 30 & 30 & 45 & 36.2667 & 3.94735 \\
\hline MTR & 30 & 1 & 4 & 2 & 0.7543 \\
\hline LTR & 30 & 1 & 3 & 1.9567 & 0.53219 \\
\hline SAR & 30 & 1.5 & 9 & 3.7167 & 1.6436 \\
\hline ENDURANCE & 30 & 14 & 48 & 29.9667 & 9.28285 \\
\hline DISABILITY & 30 & 30 & 77 & 43.9 & 13.25441 \\
\hline EWFRT & 30 & 3 & 34 & 13.2833 & 8.0694 \\
\hline Valid N (listwise) & 30 & & & & \\
\hline
\end{tabular}

Table 2: shows the correlation values among, Scapular muscle strength of UT, LT, and SA, and grip strength.

\begin{tabular}{|c|c|c|}
\hline & P value & r vale \\
\hline MTR-EWFRT & 0 & 0.931 \\
\hline LRT-EWFRT & 0 & 0.901 \\
\hline SAR-EWFRT & 0 & 0.961 \\
\hline ENDURANCE-EWFRT & 0 & 0.939 \\
\hline DISABILITY-EWFRT & 0 & 0.729 \\
\hline
\end{tabular}

Graphical representation of middle trapezius right and elbow flexion right 1.

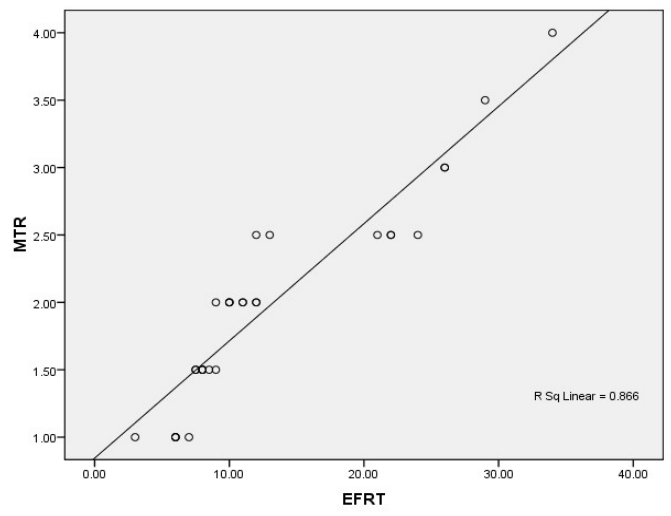

Graphical representation of lower trapezius and elbow flexion right 2.

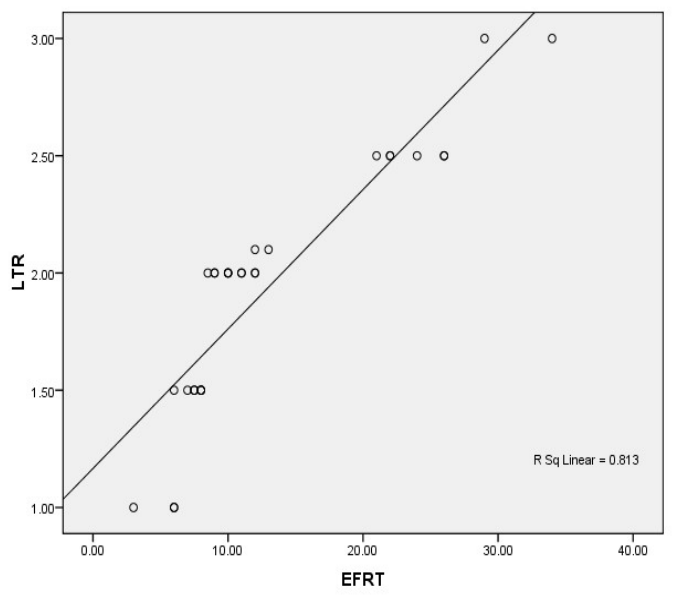

Graphical representation of serratous anterior and elbow flexion right 3.

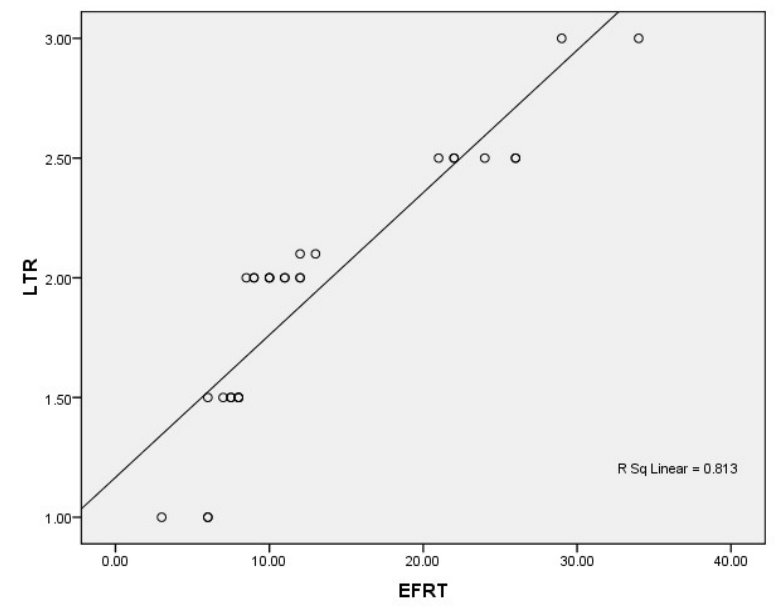

Graphical representation of pain disability and elbow flexion right 4.

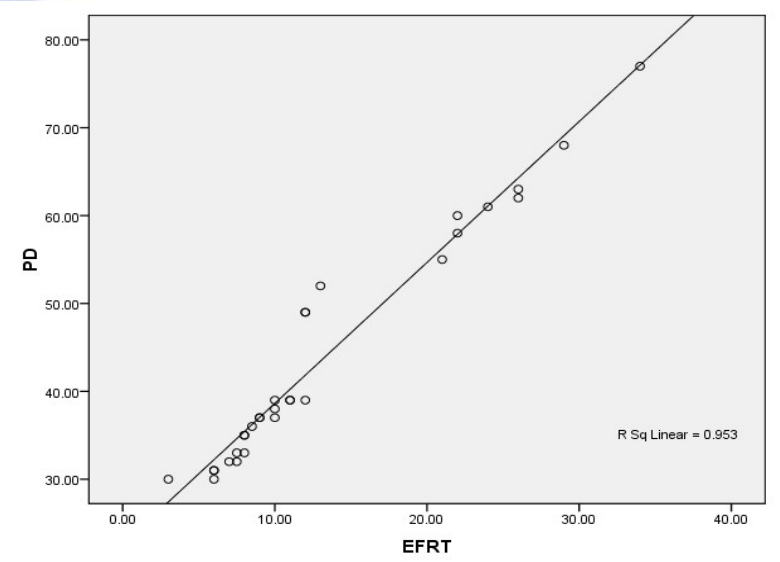

Graphical representation of endurance elbow flexion right 5.

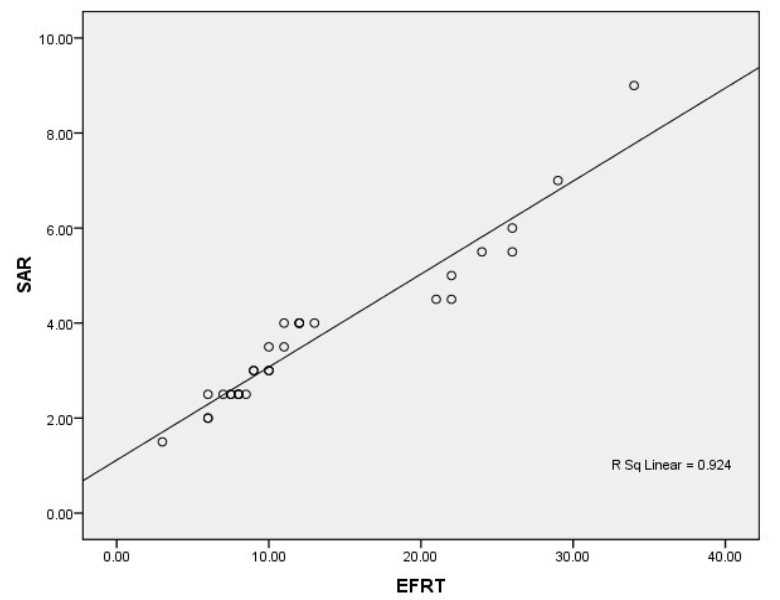

DISCUSSION

Lateral epicondylitis is an overuse injury of common extensor tendon at elbow joint. Among computer users there is common complaint in elbow and wrist region pain. It is theorized that over mechanical tension can arise in the radial nerve with sustained keyboarding due to 
sustained static work of the elbow extensor muscles [9].

Samani A study showed that pain at the upper trapezius (UT) produces an increase in wrist extensor electromyographic (EMG) signal in healthy individuals, which could potentially lead to overuse injury, such as LE, at the elbow [10]. The present study assessed scapular muscle strength using push pull dynamometer and scapular muscle endurance assessed by the using A cuff weight of $1 \%$ of the par-ticipant's body weight was strapped just proximal to the el-bow jont and grip strength was measured by using hand held dynamometer.

The age group of the present study was taken as 25-45years.in computer operators with both gender with male $27 \%$ and female $73 \%$. Assendelft $\mathrm{W}$ et al study result showed that the prevalence of Lateral Epicondylitis is more in those individuals whose sitting time with computer at least 4-6 hours per day. Right side elbow was dominantly involved in all respondents and another study showed that female respondents (67.9\%) were more as compared to male (32.1\%). Age group of computer users was 1845 years. Among respondents' students, office workers and bankers were included. More respondents use computer from last 4-6(33.11\%) and $7-9$ (23.65\%) hours. Students (54.05 \%) response was more as compared to office workers (13.85\%) and bankers (32.09\%). These values were similar with previous studies which showed that location of symptoms was more prominent $(58.45 \%)$ in those individual who use computer 4-6 hours/day [11].

The present study correlated between scapular muscle performance and lateral epicondylitis, and grip strength. There was a strong positive correlation between scapular muscle performance and lateral epicondylities The correlation values between MTR,UTR, and SAR strength and scapular muscle endurance with hand grip strength in elbow flexion position $r$ vlues are MTR-EWFRT=931, .LRT-EWFRT $=.901$, SAREWFRT $=.961 ., . E N D U R A N C E-E W F R T=.939,$. DISABILITY-EWFRT $=.729$.

Study done by JOSEPH M. DAY showed that results scap-ular muscle performance were impaired in patients with LE compared to uninvolved limb, when comparing the patients' involved limb to their unin-volved limb, the differences, although sta-tistically significant and only for SA and LT strength. The study is also demonstrates diminished scapular muscle endurance in patients with LE [7].

Erik R. Dorf, study showed that Grip strength was no different in flexion and extension for the healthy extremity and $29 \%$ stronger in flexion than in extension for the lateral epicondylitis [8].

DeSmet, Fabry, and Lieber, showed that patients who had LE by physical examination findings are likely to have significant differences between the Gs-F and the Gs-E.

The present study showed that there is an impairment of scapular muscle performance grip strength in lateral epcondylitis among computer operators.

Study has several limitations firstly we did not considered the posture like rounded posture and forward head posture, and chronic lateral epicondylitis, large age group and sample.

\section{CONCLUSION}

Present study concluded that there is a strong positive correlation between scapular muscle performance and grip strength among computer operators with lateral epicondylits.

\section{ABBREVIATIONS}

LE - lateral epicondylitis

MT - Middle trapezius

LT - Lower trapezius

SA - Serratous anterior

EWFR - Elbow flexion right

MTR - Middle trapezius right

LTR - Lower trapezius right

\section{ACKNOWLEDGEMENTS:}

I would like to acknowledge my Principal and entire staff members who helped me to complete this work with adequate instruction. My sincere thanks to my guide Dr Priya $S$, for the endless guidance and support have been pivotal for completing my paper. I express my sincere thanks to my close friends who helped me by providing valuable information to carry out my work.

\section{Conflicts of interest: None}




\section{REFERENCES}

[1]. Joseph M, Day,PT, Heather Bush.Arthur J.Nitz,Timl.Uhl. Scapular muscle performance in individual with lateral epicondylities.journals oforhopedic and sports physical therapy. 2015;45(5).

[2]. Samani A, Fernández-Carnero J, Arendt-Nielsen L, Madeleine P. Interactive effects of acute experimental pain in trapezius and sored wrist extensor on the electromyography of the fore-arm muscles during computer work. Appl Ergon. 2011;42:735-740.

[3]. Lucado AM, Kolber MJ, Cheng MS, Echternach Sr JL. Upper extremity strength characteristics in female recreational tennis players with and without lateral epicondylalgia. journal of orthopaedic \& sports physical therapy. 2012 Dec;42(12):1025-31.

[4]. Coombes BK, Bisset L, Vicenzino B. Management of lateral elbow tendinopathy: one size does not fit all. journal of orthopaedic \& sports physical therapy. 2015 Nov;45(11):938-49.

[5]. Melek Ardahan' Hatice Simsek ,Analyzing musculoskeletal system discomforts and risk factors in computer-using office workers.Pak J Med Sci. 2016 Nov-Dec;32(6):1425-1429.

[6]. Ju-hyun Lee, Tae-ho Kim, Kyu-bong Lim. Effects of eccentric control exercise for wrist extensor and shoulder stabilization exercise on the pain and functions of tennis elbow. J Phys Ther Sci. 2018 Apr; 30(4): 590-594.
[7]. Day JM, Bush H, Nitz AJ, Uhl TL. Scapular muscle performance in individuals with lateral epicondylalgia. journal of orthopaedic \& sports physical therapy. 2015 May;45(5):414-24.

[8]. Dorf ER. Chhabra AB, Golish SR, McGinty $\mathrm{JL}$, Pannunzio ME. Effect of elbow position on grip strength in the evaluation of lateral epicondylitis. J Hand Surg Am. 2007 Jul-Aug;32(6):882-6.

[9]. Vanitha Arumugam,'Senthil Selvam,'Joy C MacDermid . Radial Nerve Mobilization Reduces Lateral Elbow Pain and Provides Short-Term Relief in Computer Users $^{\S}$. Open Orthop J. 2014; 8: 368-371.

[10]. Samani A, Fernández-Carnero J, Arendt-Nielsen. Interactive effects of acute experimental pain in trapezius and sored wrist extensor on the electromyography of the fore-arm muscles during computer work. Appl Ergon. 2011;42:735-740.

[11]. Samuel A. Taylor, Jo A. Hannafin .Evaluation and Management of Elbow Tendinopathy Sports Health. 2012 Sep; 4(5): 384-393.

How to cite this article:

Priya S, Harish S Krishna, Theertha K, Salbin Sebastian. RELATIONSHIP BETWEEN SCAPULAR MUSCLE PERFORMANCE AND GRIP STRENGTH IN LATERAL EPICONDYLITIS AMONG COMPUTER OPERATORS. Int J Physiother Res 2019;7(6):3275-3280. DOI: 10.16965/ijpr.2019.170 